\title{
Iterative Reconstruction for Increased Imaging Throughput in Lab-based 3D XRM
}

\author{
Matthew Andrew ${ }^{1, *}$, William Thompson ${ }^{1}$, Arjun Kumar $^{1}$ and Benjamin Hornberger ${ }^{1}$ \\ 1. Carl Zeiss Microscopy, Pleasanton, CA, USA \\ * Corresponding author, matthew.andrew@zeiss.com
}

Imaging throughput, the number of datasets that can be acquired in a given amount of time, is one of the challenges of laboratory-based 3D X-ray Microscopy (XRM). Acquisition times for high resolution datasets can be hours, which can make the economic case difficult for industrial applications, but also in academia where laboratories need to support their operational costs by charging for instrument access. Furthermore, for in situ experiments, where multiple datasets are acquired under varying conditions, data acquisition times limit the temporal resolution achievable and the number of data points that can practically be collected. A typical workflow consists of sample preparation, scan setup, data acquisition, 3D reconstruction, image processing/segmentation, and analysis. In repetitive workflows, where many similar samples are imaged under equivalent conditions, data acquisition is typically the slowest step.

Traditionally, 3D XRM, or computed tomography (CT), uses analytical algorithms to reconstruct the 3D volume from a set of $2 \mathrm{D}$ projections acquired over a range of angular directions. The most common type is filtered back projection (for cone-beam setups known as FDK [1]). Here, the entire volume is reconstructed in a single step by Fourier-filtering the projections and projecting them all back along their respective directions. This approach is computationally simple and does not require detailed assumptions about the image formation process or the sample. However, it is prone to artifacts and the impact of noise, so to achieve good results it requires low noise data acquired at sufficiently finely spaced angular intervals (typ. 1,600 projections over 360 degrees for a $1024^{2}$ pixel detector). Iterative algorithms [e.g., 2] are more novel developments with the potential to overcome various limitations of analytical algorithms. Here, the 3D volume is built up sequentially over several iterations. At each iteration, the existing volume is forward-projected, these projections are compared to the original projections, and the difference is added to the volume. In the process, assumptions about the image formation process (e.g., noise models) as well as about the sample can be integrated to achieve a more faithful representation of the sample. Thereby, iterative algorithms can potentially achieve superior results compared to analytical algorithms, e.g. by speeding up data acquisition or reducing artifacts.

Challenges to the application of iterative algorithms include computational demands, user expertise requirements to properly tune the parameters, and sample specificity. In this paper we demonstrate results of a novel iterative algorithm [3] applied to 3D XRM of carbonate rock. We demonstrate that the iterative algorithm can achieve equivalent or superior image quality compared to FDK (measured in terms of signal-to-noise ratio, or SNR, and edge sharpness) with one-quarter of the number of projections. At the same time, the implementation is efficient (tomographic reconstruction time is about 3 minutes on a high performance, dual-GPU workstation) and user-friendly.

A carbonate rock sample of $10 \mathrm{~mm}$ diameter was imaged using 1601 projections on a ZEISS Xradia 520 Versa microscope $(10 \mu \mathrm{m}$ voxels, total acquisition time 1.5 hours). The full dataset of 1601 projections, and a subset of 401 projections (representative of 1/4 of the acquisition time), were reconstructed using both FDK and the iterative algorithm. The FDK reconstruction from 401 projections was additionally processed using an NLM, or non-local means (edge preserving noise reduction) filter. SNR was determined from two regions of interest (ROIs), one located within the rock phase and the 
other located within the pore phase. Mean and standard deviation of the two ROIs were calculated. SNR is then given by the difference of the mean values (signal) divided by the average of the standard deviations (noise). Edge sharpness was determined by fitting a logistics function across a line profile crossing a pore/grain interface. Edge sharpness, in voxels, is given by the transition width of the fitted curve (1/k parameter of the logistics function) [3]. Example images, and the resulting SNR and edge sharpness values, are shown in Fig. 1. In summary, iterative reconstruction at 401 projections produces results superior to FDK at 1601 projections (SNR 75\% higher, edge 30\% sharper). FDK at 401 projections is noisy and exhibits substantial sampling artifacts that cannot be entirely removed by the NLM filter. For iterative reconstruction at 1601 projections, the measured values are still better than at 401 projections (and FDK at 1601), although visually the quality difference is less noticeable.

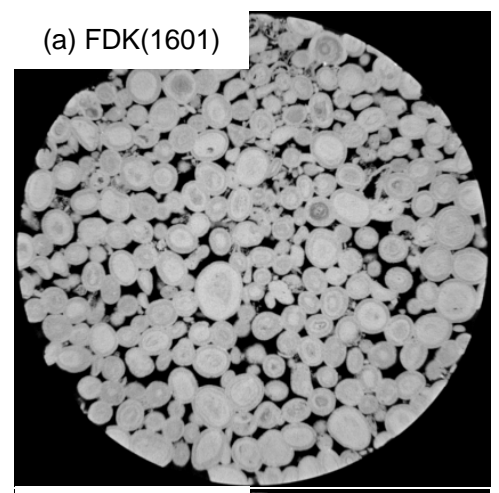

(d) $\operatorname{ITER}(1601)$

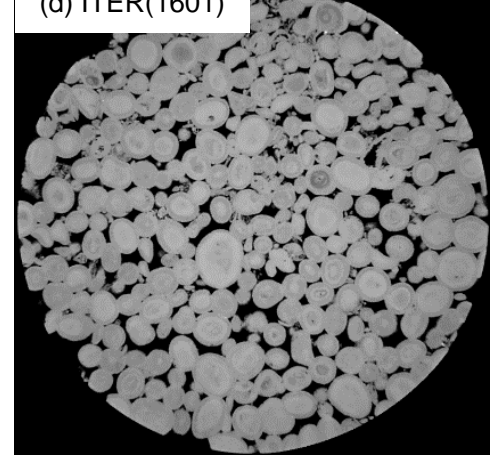

(b) $\operatorname{FDK}(401)$

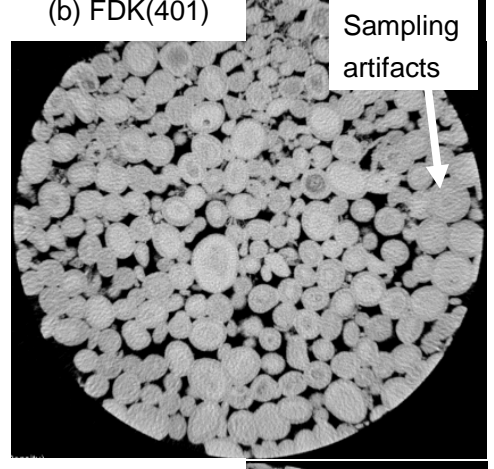

(e) ITER(401)

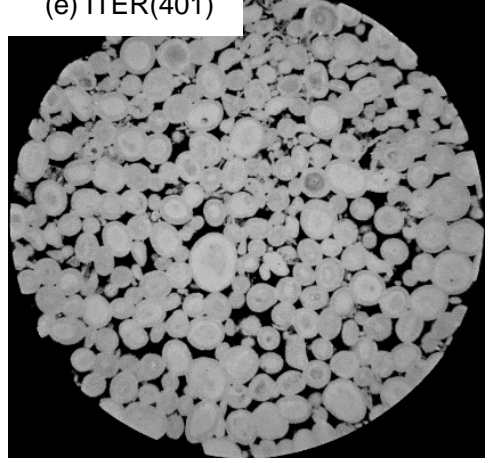



(f)
Sampling artifacts

reduced but still

present

\begin{tabular}{lrr} 
& SNR $^{(1)}$ & $\begin{array}{r}\text { Edge sharpness } \\
\text { (voxels) }^{(2)}\end{array}$ \\
\hline FDK(1601) & 39.1 & 4.3 \\
FDK(401) & 17.4 & 4.0 \\
FDK(401)+NLM & 55.5 & 4.1 \\
ITER(1601) & 88.9 & 2.3 \\
ITER(401) & 67.9 & 2.6 \\
\hline
\end{tabular}

(1) Higher is better, (2) lower is better

Figure 1: (a-e) Example slices of the 3D volume reconstructed with FDK or iterative algorithm (ITER). The number of projections in each case is labeled. (f) Summary of Signal-to-Noise and edge sharpness.

In conclusion, we have demonstrated that iterative algorithms can generate results equivalent or superior to traditional analytical algorithms with substantially shorter data acquisition time. This allows increasing imaging throughput in lab-based 3D XRM. The algorithm presented here has shown to work well for samples with sufficient "structural sparsity", i.e. features such as grains and pores are large relative to the voxel size. Future developments can extend the algorithm to a broader range of samples or address specific challenges such as limited-angle artifacts when imaging large, flat samples.

\section{References:}

[1] LA Feldkamp, LC Davis and JW Kress, J. Opt. Soc. Am. A. 1 (1984), p. 612

[2] J Fessler in "Handb. Med. Imaging Vol. 2. Med. Image Process. Anal.”, ed. JM Fitzpatrick and Milan Sonka, (SPIE Digital Library), p. 1

[3] Q Lin et al, Adv. Water Resour. (2018), in press. DOI:10.1016/j.advwatres.2018.03.007 